\title{
Transient prehypertensive treatment in spontaneously hypertensive rats: A comparison of losartan and amlodipine regarding long-term blood pressure, cardiac and renal protection
}

\author{
FENG PENG, JINXIU LIN, LIMING LIN and HONG TANG \\ The First Clinical Medical College of Fujian Medical University, Fuzhou 350005, P.R. China
}

Received July 7, 2012; Accepted August 21, 2012

DOI: $10.3892 /$ ijmm.2012.1153

\begin{abstract}
The aim of this study was to compare the effectiveness of transient prehypertensive treatment with losartan compared with amlodipine in spontaneously hypertensive rats (SHRs) on long-term blood pressure (BP), cardiac and renal protection. SHRs were prehypertensively treated with losartan, amlodipine or saline. Rats were followed up until 46 weeks of age. The left ventricular (LV) geometry and function were assessed by echocardiography. Angiotensin II (Ang II) and aldosterone (Aldo) were measured by radioimmunoassay. Ang II type $1\left(\mathrm{AT}_{1} \mathrm{R}\right)$ and type $2\left(\mathrm{AT}_{2} \mathrm{R}\right)$ receptor protein expression was determined by western blotting. The systolic blood pressure (SBP) in losartan-treated SHRs (SHR-Los) was persistently reduced until 46 weeks of age, but returned to untreated SHR levels in amlodipine-treated SHRs (SHR-Aml) from 30 weeks onwards. Compared to untreated SHRs, the albuminuria excretion in SHR-Los at week 46 was markedly decreased, the plasma, myocardium and renal tissue Ang II and Aldo levels in SHR-Los at week 46 were markedly decreased; $\mathrm{AT}_{1} \mathrm{R}$ and TGF- $\beta_{1}$ protein expression was downregulated and $\mathrm{AT}_{2} \mathrm{R}$ protein was upregulated. Compared to untreated SHRs, the left ventricular mass index (LVMI) and collagen volume fraction (CVF) in SHR-Los were markedly decreased until week 46 , and the left ventricular ejection fraction (LVEF) and cardiac brain natriuretic peptide mRNA expression were improved, whereas similar LVMI and elevated CVF were observed in SHR-Aml, and the LVEF decreased significantly below that of untreated SHRs at week 46, with cardiac BNP mRNA expression increasing slightly. Prehypertensive treatment with losartan was more effective than amlodipine on delaying long-term BP increase
\end{abstract}

Correspondence to: Dr Jinxiu Lin, The First Clinical Medical College of Fujian Medical University, Fuzhou 350005, P.R. China E-mail: jinxiulincn@126.com

Key words: prehypertensive treatment, spontaneously hypertensive rats, losartan, amlodipine, blood pressure, renin-angiotensin system and ameliorating cardiac, renal structure and function, which may be related to the permanent attenuation of the circulating and local renin-angiotensin systems.

\section{Introduction}

Evidence has demonstrated that individuals with prehypertension are at an increased risk of developing hypertension and suffering cardiovascular events, poor cognitive performance, type 2 diabetes, as well as kidney damage (1-5). Lifestyle modification is recommended as a basis for all subjects belonging to this blood pressure (BP) category by current guidelines for hypertension, and drug therapy is considered for individuals with hypertension concomitant with diabetes or kidney disease when a trial of lifestyle modification fails to reduce their BP to $130 / 80 \mathrm{mmHg}$ or less (6). However, poor adherence makes its implementation into clinical settings difficult and relatively ineffective. Recently published TROPHY and PHARAO trials demonstrated that prehypertensive treatment with a renin-angiotensin system (RAS) inhibitor over a relatively short time reduces the risk of incident hypertension and is well tolerated $(7,8)$. In addition, studies using spontaneously hypertensive rats (SHRs) also indicate that early and transient treatment with an angiotensinconverting-enzyme inhibitor (ACEI), angiotensin-receptor blockers (ARB) or aldosterone (Aldo) antagonist attenuates the development of hypertension and exerts cardiovascular protective effects up to an advanced age (9-11).

The aforementioned studies suggest that the reninangiotensin-Aldo system (RAAS) blockade may be a feasible and beneficial option for individuals with prehypertension, which raises the question whether the effects are restricted only to RAAS antagonists or whether they may also be extended to other forms of therapies, such as calcium channel blockers (CCBs). To our knowledge, this question remains unanswered.

CCBs are a heterogeneous class of drugs used in the treatment of coronary heart disease and hypertension. Amlodipine, a third-generation dihydropyridine CCB, is characterized by high vascular selectivity and relatively low negative inotropic effects. Previous studies involving animal models have proven that amlodipine is effective in preventing hypertension-related left ventricular hypertrophy (LVH) and dysfunction $(12,13)$. 
However, research concerned with its use in the prehypertensive stage remains limited.

In the present study, we aimed to compare the effectiveness of prehypertensive treatment with losartan compared with amlodipine in SHRs on long-term BP, renal and cardiac protection, and to investigate the underlying mechanism of their effects.

\section{Materials and methods}

Animals and pharmacological treatment. A total of 72 four-week-old male SHRs (purchased from Shanghai Slac Laboratory Animal Co., Ltd.) were randomly divided into 3 groups and were administered losartan [losartan-treated SHRs (SHR-Los), $20 \mathrm{mg} / \mathrm{kg} / \mathrm{day}, \mathrm{n}=24$ ], amlodipine [amlodipine-treated SHRs (SHR-Aml), $10 \mathrm{mg} / \mathrm{kg} / \mathrm{day}, \mathrm{n}=24]$ or saline (SHRs, $\mathrm{n}=24$ ) by gavage for 6 weeks. Age- and gendermatched Wistar-Kyoto rats (WKYs, n=24) served as the normal control. Rats were housed 5/cage under controlled temperature $\left(22 \pm 1^{\circ} \mathrm{C}\right)$ and humidity $(60 \%)$ conditions, and were exposed to a 12-h dark-light cycle. Standard food and tap water were supplied ad libitum. All experiments were approved by the Animal Ethics Committee of Fujian Medical University and performed in accordance with institutional guidelines.

Experimental protocol. Rats were followed up until week 46. Systolic blood pressure (SBP) was measured at weeks 4 and 10 and monthly after treatment was stopped. Cardiac, renal structure and function, circulating and local RAS components were repeatedly assessed at weeks 14,30 and 46, with 8 rats sacrificed/group at each time point for analysis. Cardiac, renal Ang II type 1 receptor $\left(A_{1} R\right)$ and type 2 receptor $\left(A_{2} R\right)$ protein were determined at weeks 14 and 46. Cardiac brain natriuretic peptide (BNP) mRNA was determined at week 46.

Blood pressure determinations. SBP was measured noninvasively in unanesthetized rats by the tail-cuff method using a specialized pressure transducer (PowerLab ML125/R NIBP System; ADInstruments) as previously described (14). SBP was determined when the cuff pressure corresponded to the restoration of the first caudal artery pulse. Three consecutive readings were averaged for each determination.

Evaluation of cardiac hypertrophy and fibrosis. Under anesthesia with chloral hydrate $(300 \mathrm{mg} / 100 \mathrm{~g}$, i.p.), the animals were sacrificed and the hearts were removed and blotted dry, the left ventricle (LV) was carefully dissected and weighed, and the left ventricular mass index (LVMI) (LV weight/100 g body weight) was calculated to indicate cardiac hypertrophy. Then, the hearts were further incubated for $24 \mathrm{~h}$ in formalin, embedded in paraffin and cut into $4-\mu \mathrm{m}$ sections for Sirius Red staining. Sections were carefully scanned with a light microscope (Olympus, Japan) connected to a computer using the Image Pro Plus 6.0 software for histomorphometry at a magnification of $x 100$. The collagen volume fraction (CVF), an index of cardiac fibrosis, was determined as the percentage of the Sirius Red-stained area/total myocardial tissue area.

Echocardiography. Transthoracic echocardiography was performed on the animals in the left lateral decubitus position, after being anesthetized with chloral hydrate $(300 \mathrm{mg} / 100 \mathrm{~g}$, i.p.). A vivid 7 echocardiographic system (GE Healthcare, USA) with a $10-\mathrm{MHz}$ transducer was used to obtain an M-mode echocardiogram from the long axis view of the LV. The left ventricular end-diastolic dimension (LVEDD), end-diastolic interventricular septum thickness (IVSTd) and left ventricular ejection fraction (LVEF) were measured following the guidelines of the American Society of Echocardiography (15).

24-h urinary albumin and creatinine clearance rate. The 24-h urine excretion was collected in metabolic cages. These studies were performed during the same weeks as the echocardiography. Urinary albumin was measured by immnonephelometric analysis (BN-II analyzer; Dade Behring, Eschborn, Germany). Creatine in serum and urine were measured by the sarcosine oxidase enzymic method using an automatic Biochemistry Analyzer (AU2700; Olympus) to calculate the creatinine clearance rate $(\mathrm{CCr})$ with the standard formula: $\mathrm{CCr}=$ (urinary creatinine x 24-h urine flow)/(serum creatinine x 1440) $\mathrm{mg} / \mathrm{min}$.

Renal histology. The kidneys were removed from the animals under pentobarbital sodium anesthesia, fixed in $4 \%$ formalin and embedded in paraffin. Central cross-sections of the whole kidney including the cortex and medulla were prepared for examination. The glomerulosclerosis index (GSI) was measured in 4- $\mu \mathrm{m}$ sections, stained with periodic acid Schiff. GSI was calculated in both superficial and juxtamedullary glomeruli for the whole interstitial area with a light microscope (Olympus) by a nephropathologist in a blinded manner using a cumulative semi-quantitative scoring as previously described (16).

Plasma, renal cortex tissue and myocardium angiotensin II (Ang II) and Aldo. The heart and renal cortex tissues were weighed $(100 \mathrm{mg})$, boiled and homogenized by Polytron in $1 \mathrm{ml}$ PBS, respectively. The homogenate was centrifuged at $3,500 \mathrm{rpm}$ for $10 \mathrm{~min}$. The tissue supernatant and plasma Ang II and Aldo levels were determined by radioimmunoassay using a commercially available kit (Beijing North Institute of Biological Technology, Beijing, China).

Western blot analysis for $A T_{1} R$ and $A T_{2} R$ proteins. Western blot analysis was performed as described by $\mathrm{Xu}$ et al (17) Briefly, LV tissue $(10 \mathrm{mg})$ and renal cortex tissue $(10 \mathrm{mg})$ were electrophoresed on SDS-polyacrylamide gels under reducing conditions, respectively. Proteins were transferred onto nitrocellulose membranes, blocked with $5 \%$ non-fat milk in TBS containing $0.05 \%$ Tween-20 (TBST) and then incubated with an anti- $\mathrm{AT}_{1} \mathrm{R}$ antibody (1:500, ab9391; Abcam), anti-AT $\mathrm{R}_{2}$ antibody (1:800, ab19134; Abcam) or anti-actin antibody (1:1000, sc-1616; Santa Cruz Biotechnology, Inc.) overnight at $4^{\circ} \mathrm{C}$. After 5 washes with TBST and 2 washes with TBS, the membranes were incubated for $1 \mathrm{~h}$ at room temperature with horseradish peroxidase-conjugated secondary antibody. Following another 2 washes with TBST, labeled proteins were visualized using ECL (sc-2048; Santa Cruz Biotechnology, Inc.) on high-performance chemiluminescence film. The intensity of the bands was quantified by densitometry with image analysis software. Results for $\mathrm{AT}_{1} \mathrm{R}$ and $\mathrm{AT}_{2} \mathrm{R}$ were expressed as a ratio of $\mathrm{AT}_{1} \mathrm{R}$ or $\mathrm{AT}_{2} \mathrm{R}$ over $\beta$-actin. 
Table I. Primer sequences.

\begin{tabular}{lllcc}
\hline Primer & \multicolumn{1}{c}{ Sequence 5' to 3' } & Annealing temperature $\left({ }^{\circ} \mathrm{C}\right)$ & Amplification product $(\mathrm{bp})$ \\
\hline BNP & Up & GCTGCTTTGGGCAGAAGATAGA & 59 & 169 \\
& Down & ACAACCTCAGCCCGTCACA & & 468 \\
GAPDH & Up & CAACGGCACAGTCAAGG & 55 & \\
& Down & CCAGTGGATGCAGGGAT & & \\
\hline
\end{tabular}

BNP, brain natriuretic peptide; GAPDH, glyceraldehyde phosphate dehydrogenase.

Quantification of cardiac BNP $m R N A$. Total RNA from ventricular tissue was isolated with TRIzol reagent (Invitrogen), and the concentration was determined by measuring the absorbance at $260 \mathrm{~nm}$. The RNA was transcribed into cDNA using M-MLV reverse transcriptase (Promega) and amplified (Takara PCR Amplification kit) using primers (Table I) specific to each gene of interest. The cycle profiles were programed as follows: initial 5-min denaturation at $95^{\circ} \mathrm{C}$ followed by 35 cycles of denaturation at $95^{\circ} \mathrm{C}$ for $30 \mathrm{sec}$, annealing at 59 or $55^{\circ} \mathrm{C}$ (for BNP and GAPDH, respectively) for $30 \mathrm{sec}$ and extension at $72^{\circ} \mathrm{C}$ for $30 \mathrm{sec}$, with a final extension at $72^{\circ} \mathrm{C}$ for $5 \mathrm{~min}$. PCR products were analyzed by $1.5 \%$ agarose gel electrophoresis. The photodensity of amplified cDNA product bands was quantified by densitometry. The BNP mRNA abundance was defined as a ratio to GAPDH mRNA.

Statistical analysis. All analyses were performed with SPSS software version 13.0 (Chicago, IL, USA). Continuous data are presented as the means \pm standard deviation (SD). Differences between groups were compared by one-way analysis of variance, followed by LSD t-test for multiple comparisons. $\mathrm{P}<0.05$ values were considered to indicate a statistically significant difference. For all tests, a two-tailed $\mathrm{P}$-value $<0.05$ was considered to indicate a significant result.

\section{Results}

Systolic blood pressure. At week 10, the SBP in the untreated SHRs was significantly higher compared to that in the WKYs (SHRs vs. WKYs: $170 \pm 5$ vs. $116 \pm 8 \mathrm{mmHg}, \mathrm{P}<0.05$ ). Prehypertensive treatment with losatran and amlodipine equally reduced BP to a midway level between untreated SHRs and WKYs (SHR-Los vs. SHR-Aml: $126 \pm 6$ vs. $124 \pm 7 \mathrm{mmHg}$; $\mathrm{P}>0.05$ ). After drug withdrawal, the SBP in SHR-Los remained significantly lower compared to that in untreated SHRs until week $46(\mathrm{P}<0.05)$, whereas SBP gradually increased to untreated SHR levels in SHR-Aml from 30 weeks onwards $(\mathrm{P}>0.05)$ (Fig. 1).

Evaluation of cardiac hypertrophy and fibrosis. Cardiac hypertrophy and fibrosis, expressed by the left ventricular hypertrophy index and myocardial CVF, respectively, were both significantly elevated in untreated SHRs compared with WKYs throughout the study $(\mathrm{P}<0.05)$. The LVMI and CVF in SHR-Los displayed similar values compared to those in WKYs at week 14 (LVMI,

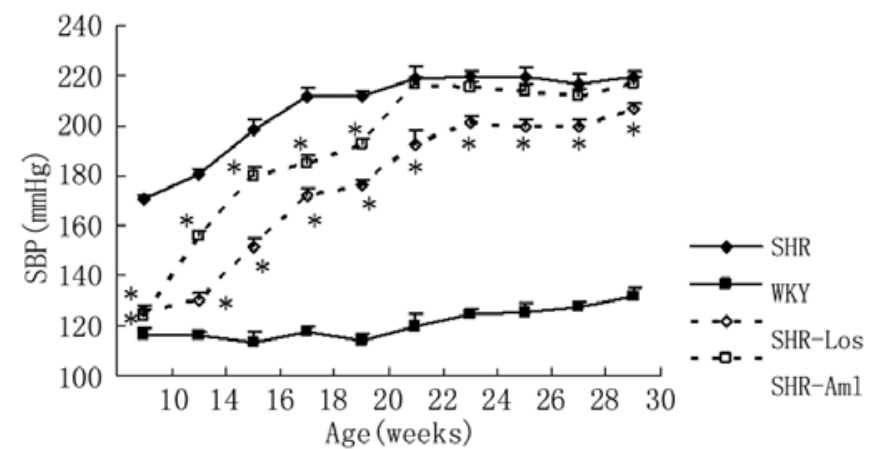

Figure 1. Comparison of SBP development in losartan-treated and amlodipine-treated SHRs after the discontinuation of prehypertensive treatment. SBP, systolic blood pressure. ${ }^{*} \mathrm{P}<0.05$ vs. SHRs at the same age.

$2.29 \pm 0.34$ vs. $2.33 \pm 0.21 \mathrm{mg} / \mathrm{g}$; CVF, $2.6 \pm 0.7$ vs. $2.6 \pm 0.4 \%$, both $\mathrm{P}>0.05$ ) and remained midway between untreated SHRs and WKYs at weeks 30 and $46(\mathrm{P}<0.05)$. Unlike losartan treatment, the LVMI in SHR-Aml was not different from that in untreated SHRs at weeks 14 and $46(\mathrm{P}>0.05)$, but was slightly reduced at week $30(2.61 \pm 0.18$ vs. $3.11 \pm 0.09 \mathrm{mg} / \mathrm{g}, \mathrm{P}<0.05)$ (Fig. $2 \mathrm{~A})$; the CVF in SHR-Aml demonstrated comparable values to untreated SHRs at weeks 14 and 30 ( $P>0.05)$, but was significantly higher compared to that in untreated SHRs at week 46 (12.1 \pm 1.3 vs. $7.8 \pm 1.5 \%, \mathrm{P}<0.05)$ (Fig. $2 \mathrm{~B})$.

Echocardiography. As shown in Table II, the IVSTd in untreated SHRs was significantly higher compared to that in WKYs throughout the experiment $(\mathrm{P}<0.05)$, and the IVSTd in SHR-Los revealed similar values as in WKYs $(\mathrm{P}>0.05)$, whereas an insignificant reduction of IVSTd was observed in SHR-Aml compared with untreated SHRs $(\mathrm{P}>0.05)$. The LVEDDs in the WKY and SHR-Los groups remained stable over the follow-up period, but increased progressively in untreated SHRs and SHR-Aml at week 46 as compared to previous values. The LVEF deteriorated significantly in untreated SHRs and even more sharply in SHR-Aml as compared with WKYs at week 46 (SHR, 79.5 $\pm 1.9 \%$; SHR-Aml, 74.4 $\pm 4.3 \%$; WKY, $84.2 \pm 2.3 \%$; both $\mathrm{P}<0.05$ ), but values remained similar for the WKY and SHR-Los groups $(83.1 \pm 2.3 \%)$.

Renal state until advanced age. Compared with WKYs, 24-h albuminuria was increased in untreated SHRs and prehypertensively treated SHRs throughout the study 

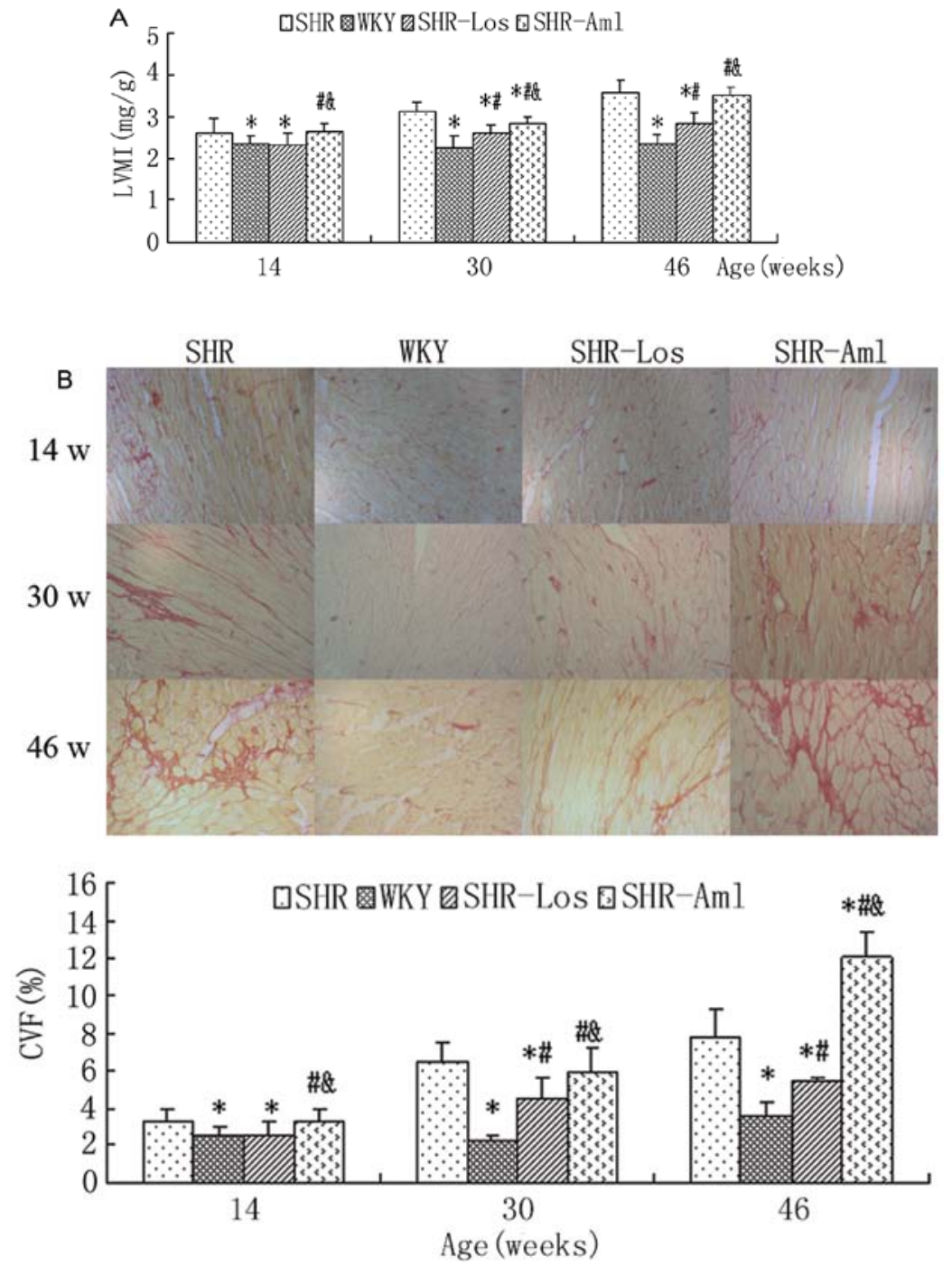

Figure 2. (A) Comparison of the long-term effects on LVMI with prehypertensive losartan and amlodipine treatment. (B) Comparison of the long-term effects on CVF with prehypertensive losartan and amlodipine treatment. Myocardial interstitial collagen appears in red and cardiac myocytes in yellow after picrosirius red staining. Original magnification, $\mathrm{x} 10 .{ }^{*} \mathrm{P}<0.05 \mathrm{vs}$. SHRs at the same age; ${ }^{*} \mathrm{P}<0.05 \mathrm{vs}$. WKYs at the same age; ${ }^{\circledR} \mathrm{P}<0.05 \mathrm{vs}$. SHR-Los at the same age. LVMI, left ventricular mass index; CVF, collagen volume fraction.

Table II. Longitudinal echocardiographic evaluation of WKYs, untreated and prehypertensively treated SHRs.

\begin{tabular}{lcccc}
\hline Group & Age (Weeks) & IVSTd (mm) & LVEDD (mm) & EF (\%) \\
\hline SHR & 14 & $2.52 \pm 0.23$ & $6.9 \pm 0.6$ & $83.2 \pm 2.9$ \\
& 30 & $2.64 \pm 0.17$ & $6.9 \pm 0.6$ & $83.5 \pm 2.7$ \\
WKY & 46 & $2.60 \pm 0.25$ & $7.6 \pm 0.7^{\mathrm{d}}$ & $79.5 \pm 1.9$ \\
& 14 & $1.72 \pm 0.07^{\mathrm{a}}$ & $7.2 \pm 0.6$ & $85.1 \pm 2.2$ \\
SHR-Los & $1.96 \pm 0.11^{\mathrm{a}}$ & $7.3 \pm 0.4$ & $85.1 \pm 1.8$ \\
& 46 & $1.92 \pm 0.22^{\mathrm{a}}$ & $7.3 \pm 0.5$ & $84.2 \pm 2.3^{\mathrm{a}}$ \\
& 14 & $1.78 \pm 0.12^{\mathrm{a}}$ & $7.2 \pm 0.9$ & $83.2 \pm 3.2$ \\
SHR-Aml & 30 & $1.85 \pm 0.13^{\mathrm{a}}$ & $7.2 \pm 0.5$ & $82.8 \pm 2.5$ \\
& 46 & $1.88 \pm 0.16^{\mathrm{a}}$ & $7.3 \pm 0.4$ & $83.1 \pm 2.3^{\mathrm{a}}$ \\
& 14 & $2.62 \pm 0.15^{\mathrm{b}, \mathrm{c}}$ & $6.8 \pm 0.7$ & $82.7 \pm 3.1$ \\
& 30 & $2.54 \pm 0.21^{\mathrm{b}, \mathrm{c}}$ & $7.0 \pm 0.3$ & $80.5 \pm 4.6$ \\
& 46 & $2.52 \pm 0.22^{\mathrm{b}, \mathrm{c}}$ & $7.8 \pm 0.7^{\mathrm{d}}$ & $74.4 \pm 4.3^{3-\mathrm{c}}$ \\
\hline
\end{tabular}

IVSTd, end-diastolic interventricular septum thickness; LVEDD, left ventricular end-diastolic dimension; EF, ejection fraction. ${ }^{a} \mathrm{P}<0.05$ vs. SHRs at the same age; ${ }^{b} \mathrm{P}<0.05$ vs. WKYs at the same age; ${ }^{\mathrm{c}} \mathrm{P}<0.05$ vs. SHR-Los at the same age; ${ }^{\mathrm{d}} \mathrm{P}<0.05$ vs. previous follow-up time. 
Table III. Longitudinal renal function evaluation of WKYs, untreated and prehypertensively treated SHRs ( $\mathrm{n}=8)$.

\begin{tabular}{lccccc}
\hline Group & $\begin{array}{c}\text { Age } \\
(\text { Weeks })\end{array}$ & $\begin{array}{c}\text { Serum creatinine } \\
(\mu \mathrm{mol} / \mathrm{l})\end{array}$ & $\begin{array}{c}\text { Serum urea nitrogen } \\
(\mathrm{mmol} / \mathrm{l})\end{array}$ & $\begin{array}{c}\mathrm{CCr} \\
(\mathrm{ml} / \mathrm{min})\end{array}$ & $\begin{array}{c}\text { Albuminuria } \\
(\mathrm{mg} / 24 \mathrm{~h})\end{array}$ \\
\hline SHR & 14 & $30.84 \pm 4.34$ & $7.79 \pm 1.00$ & $1.76 \pm 0.14$ & $11.42 \pm 2.60$ \\
& 30 & $37.80 \pm 7.09$ & $7.87 \pm 1.24$ & $1.40 \pm 0.12$ & $20.51 \pm 4.61$ \\
& 46 & $41.25 \pm 5.18$ & $8.85 \pm 1.30$ & $0.71 \pm 0.06$ & $45.46 \pm 5.55$ \\
WKY & 14 & $30.61 \pm 4.41$ & $7.17 \pm 1.16$ & $1.81 \pm 0.12$ & $10.65 \pm 1.91$ \\
& 30 & $34.53 \pm 5.78$ & $7.28 \pm 1.26$ & $1.89 \pm 0.16^{\mathrm{a}}$ & $9.24 \pm 1.40^{\mathrm{a}}$ \\
& 46 & $34.81 \pm 6.50^{\mathrm{a}}$ & $7.92 \pm 1.09$ & $1.74 \pm 0.06^{\mathrm{a}}$ & $10.27 \pm 2.19^{\mathrm{a}}$ \\
SHR-Los & 14 & $30.74 \pm 2.43$ & $7.10 \pm 1.02$ & $1.74 \pm 0.11$ & $10.44 \pm 1.40$ \\
& 30 & $34.81 \pm 8.20$ & $7.29 \pm 1.10$ & $1.71 \pm 0.13^{\mathrm{a}, \mathrm{b}}$ & $15.35 \pm 5.05^{\mathrm{a}, \mathrm{b}}$ \\
& 46 & $35.40 \pm 5.19^{\mathrm{a}}$ & $8.21 \pm 1.21$ & $1.52 \pm 0.08^{\mathrm{a}, \mathrm{b}}$ & $22.66 \pm 3.16^{\mathrm{a}, \mathrm{b}}$ \\
SHR-Aml & 14 & $31.50 \pm 3.53$ & $7.46 \pm 0.69$ & $1.79 \pm 0.13$ & $10.97 \pm 3.06$ \\
& 30 & $33.96 \pm 6.46$ & $7.56 \pm 0.70$ & $1.70 \pm 0.11^{\mathrm{a}, \mathrm{b}}$ & $18.20 \pm 3.24^{\mathrm{b}}$ \\
& 46 & $36.44 \pm 3.75$ & $8.35 \pm 1.18$ & $1.11 \pm 0.10^{\mathrm{a}-\mathrm{c}}$ & $40.92 \pm 4.48^{\mathrm{b}, \mathrm{c}}$ \\
\hline
\end{tabular}

$\mathrm{CCr}$, creatinine clearance rate; ${ }^{a} \mathrm{P}<0.05$ vs. SHRs at the same age; ${ }^{\mathrm{b}} \mathrm{P}<0.05$ vs. WKYs at the same age; ${ }^{\mathrm{c}} \mathrm{P}<0.05$ vs. SHR-Los at the same age. The data are represented as the means $\pm \mathrm{SD}$.

period. A steep increase in albumin excretion was noticed in untreated SHRs $(20.51 \pm 4.61 \mathrm{mg} / \mathrm{day}, \mathrm{P}<0.05)$ and SHR-Aml $(18.20 \pm 3.24 \mathrm{mg} / \mathrm{day}, \mathrm{P}<0.05)$ at 30 weeks of age. However, this surge was blunted in SHR-Los $(15.35 \pm 5.05 \mathrm{mg} / \mathrm{day}$, $\mathrm{P}<0.05)$. Albuminuria, however, remained significantly higher in these rats compared to WKYs $(9.24 \pm 1.40 \mathrm{mg} /$ day). At 46 weeks of age, 24-h albuminuria remained low in WKYs $(10.27 \pm 2.19 \mathrm{mg} / \mathrm{day})$, but increased further in untreated SHRs $(45.46 \pm 5.55 \mathrm{mg} / \mathrm{day}, \mathrm{P}<0.05)$ and SHR-Aml $(40.92 \pm 4.48 \mathrm{mg} / \mathrm{day}, \mathrm{P}<0.05)$, whereas the difference between the two groups with respect to albuminuria was further pronounced (SHR-Los $22.66 \pm 3.16 \mathrm{mg} /$ day, $\mathrm{P}<0.05$ ). Contrary to 24-h albuminuria, continual decline in $\mathrm{CCr}$ occurred in the untreated SHR and SHR-Aml groups throughout the study period, whereas the level was stable in WKYs. A steep increase in $\mathrm{CCr}$ was noticed in untreated SHRs $(0.71 \pm 0.06 \mathrm{ml} / \mathrm{min}, \mathrm{P}<0.05)$ and SHR-Aml $(1.11 \pm 0.10 \mathrm{ml} / \mathrm{min}$, $\mathrm{P}<0.05)$ at 46 weeks of age. However, this surge was blunted in SHR-Los $(1.52 \pm 0.08 \mathrm{ml} / \mathrm{min}, \mathrm{P}<0.05)$. The data obtained are depicted in Table III. Glomerular morphology at 14 weeks of age demonstrated enhanced sclerosis in untreated SHRs as compared with WKYs. Intermediate values were revealed in SHR-Los and SHR-Aml. A rapid progression of glomerulosclerosis occurred in the untreated SHR and SHR-Aml groups. There were no differences in SHR-Aml compared to untreated SHRs, whereas SHR-Los had a significantly attenuated glomerulosclerosis compared to both groups in 46-week-old animals. This pattern was most pronounced in juxtamedullary glomeruli (Fig. 3A and B).

Plasma, renal cortex tissue and myocardium Ang II and Aldo. The plasma Ang II in untreated SHRs was comparable to WKYs at weeks 14 and 30, but was significantly higher compared to that in WKYs at week 46. The plasma Ang II in SHR-Los and SHR-Aml were both significantly higher compared to those in untreated SHRs at week $14(\mathrm{P}<0.05)$. At weeks 30 and 46, the plasma Ang II in SHR-Los was significantly reduced compared with the untreated SHR and WKY groups $(\mathrm{P}<0.05)$, whereas the plasma Ang II in SHR-Aml exhibited similar values to untreated SHRs $(\mathrm{P}>0.05)$. The difference in plasma Aldo between untreated SHR and WKY groups was consistent with that in plasma Ang II throughout the study period. The plasma Aldo in SHR-Los was permanently attenuated compared with untreated SHRs $(\mathrm{P}<0.05)$, however, no significant difference of plasma Aldo was observed between the SHR-Aml and untreated SHR groups ( $\mathrm{P}>0.05$ ) (Fig. 4A and B). The Ang II and Aldo of renal cortex tissue and myocardium in untreated SHRs were markedly higher compared to those in WKYs throughout the follow-up period. The myocardium Ang II and Aldo in SHR-Los were significantly decreased compared with untreated SHRs $(\mathrm{P}<0.05)$, whereas no significant difference of myocardium Ang II and Aldo was observed between the SHR-Aml and untreated SHR groups ( $>>0.05$ ) (Fig. 4C and D). The renal cortex tissue Ang II and Aldo in SHR-Los were significantly decreased compared with untreated SHRs $(\mathrm{P}<0.05)$, whereas no significant difference of renal cortex tissue Ang II and Aldo was observed between the SHR-Aml and untreated SHR groups ( $\mathrm{P}>0.05)$ (Fig. 4E and $\mathrm{F}$ and Table IV).

Western blot analysis for $A T_{1} R$ and $A T_{2} R$ proteins. At weeks 14 and 46 , the cardiac $\mathrm{AT}_{1} \mathrm{R}$ protein expression in untreated SHRs was significantly higher compared to that in WKYs $(\mathrm{P}<0.05)$, and the reverse occurred for the $\mathrm{AT}_{2} \mathrm{R}$ protein $(\mathrm{P}<0.05)$ (Fig. 5). The $\mathrm{AT}_{1} \mathrm{R}$ protein expression was downregulated while the $\mathrm{AT}_{2} \mathrm{R}$ protein was upregulated in the SHR-Los group as compared with untreated SHRs; no significant difference of the two protein levels was observed between the SHR-Aml and untreated SHR groups $(\mathrm{P}>0.05)$. The renal $\mathrm{AT}_{1} \mathrm{R}$ and $A T_{2} \mathrm{R}$ protein expression levels were 

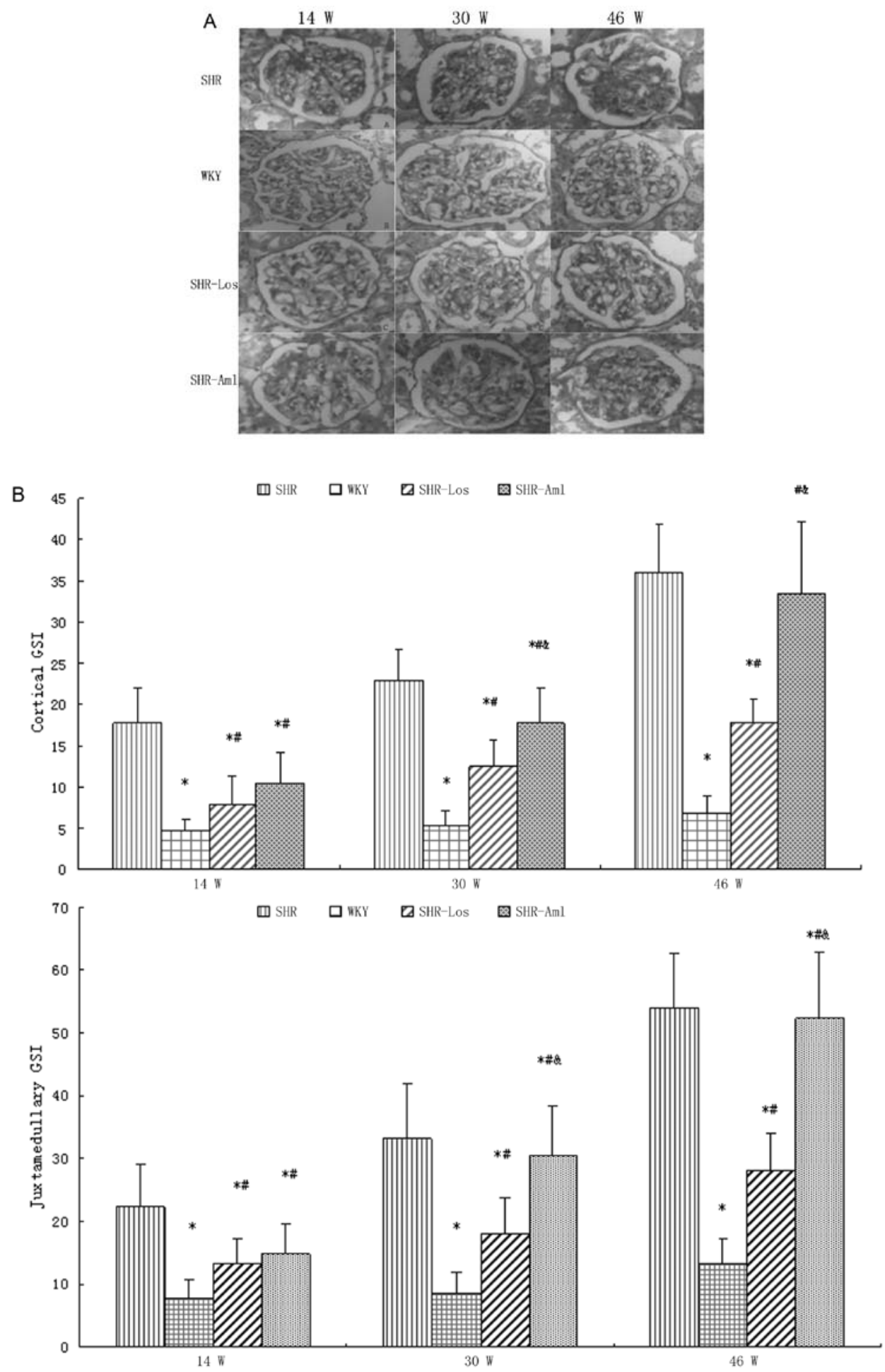

Figure 3. (A) Sections from rat kidneys with H\&E staining. H\&E-stained sections were evaluated by a veterinary pathologist who was blinded to the experimental conditions. The SHR group demonstrated a proliferation of mesangial cells and matrix, an enlargement of the mesangial region, glomerulosclerosis and a compressive deformation of capillary loops. The SHR-Los group demonstrated a significantly attenuated glomerulosclerosis compared to the SHR group. Treatment with losartan prevented the development of alterations. The SHR-Los group attenuated deposit of matrix, an inconspicuous enlargement of the mesangial region or proliferation of mesangial cells. A rapid progression of glomerulosclerosis occurred in the SHR-Aml group. All images were captured at a magnification of $\mathrm{x} 400$. (B) Influence of losartan and amlodipine on glomerulosclerosis index (GSI). Values represent the means $\pm \mathrm{SD}, \mathrm{n}=8 / \mathrm{group}$ for analysis. ${ }^{*} \mathrm{P}<0.05$ vs. SHRs at the same age; ${ }^{\prime} \mathrm{P}<0.05$ vs. WKYs at the same age; ${ }^{\circledR} \mathrm{P}<0.05$ vs. SHR-Los at the same age.

similar to the results of the cardiac levels at weeks 14 and 46 , and TGF- $\beta_{1}$ protein expression in untreated SHRs was significantly higher compared to that in WKYs $(\mathrm{P}<0.05)$. The renal TGF- $\beta_{1}$ protein expression was lower in SHR-Los as compared with untreated SHRs, and no significant difference of the two protein levels was observed between the SHR-Aml and untreated SHR group (Fig 6).

Quantification of cardiac BNP mRNA. At week 46, the cardiac BNP mRNA expression in untreated SHRs was notably 

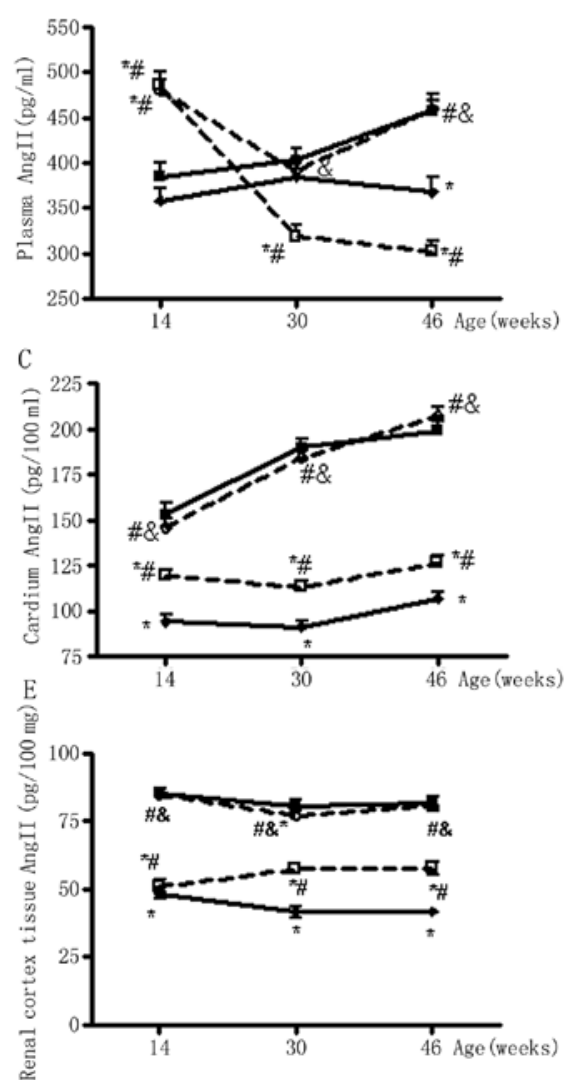

B
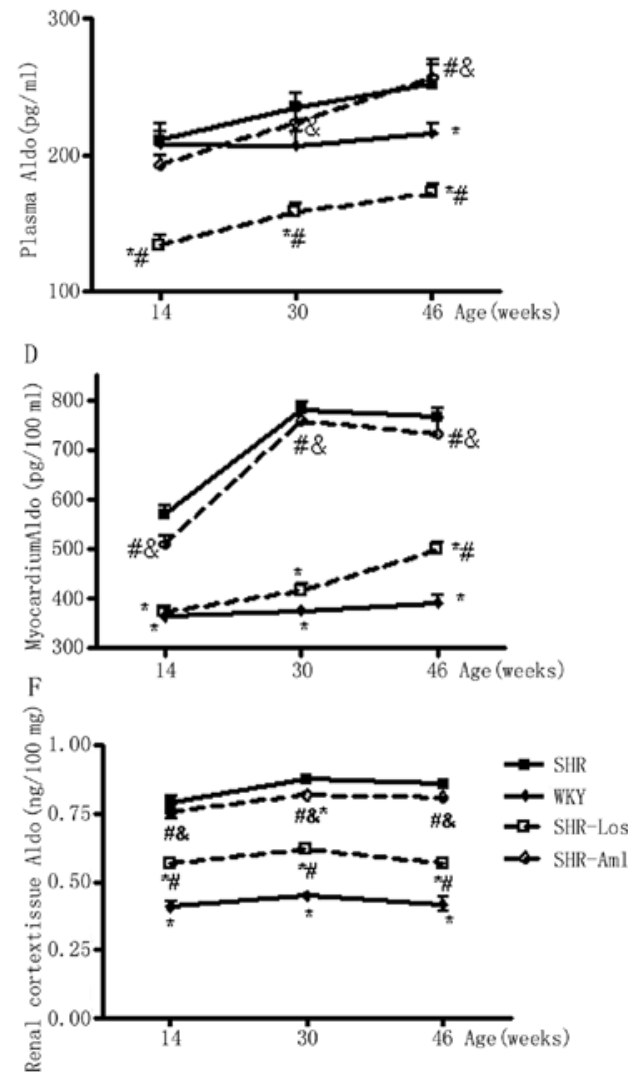

Figure 4. Comparison of the long-term effects of prehypertensive treatment with losartan and amlodipine on (A and B) plasma, (C and D) myocardium and (E and F) renal cortex tissue Ang II and Aldo levels. Ang II, angiotensin II; Aldo, aldosterone. ${ }^{*} \mathrm{P}<0.05$ vs. SHRs at the same age; "P<0.05 vs. WKYs at the same age; ${ }^{\circledR} \mathrm{P}<0.05$ vs. SHR-Los at the same age; ${ }^{\circledR} \mathrm{P}<0.05$ vs. SHR-Los at the same age.

Table IV. Evaluation of longitudinal plasma and renal cortex tissue Ang II and Aldo levels of WKYs, untreated and prehypertensively treated SHRs $(n=8)$.

\begin{tabular}{lccccc}
\hline Group & $\begin{array}{c}\text { Age } \\
(\text { Weeks })\end{array}$ & $\begin{array}{c}\text { Plasma Ang II } \\
(\mathrm{pg} / \mathrm{ml})\end{array}$ & $\begin{array}{c}\text { Plasma Aldo } \\
(\mathrm{ng} / \mathrm{ml})\end{array}$ & $\begin{array}{c}\text { Renal cortex tissue Ang II } \\
(\mathrm{pg} / 100 \mathrm{mg})\end{array}$ & $\begin{array}{c}\text { Renal cortex tissue Ang II } \\
(\mathrm{ng} / 100 \mathrm{mg})\end{array}$ \\
\hline SHR & 14 & $385.02 \pm 45.24$ & $0.21 \pm 0.033$ & $85.15 \pm 5.53$ & $0.79 \pm 0.076$ \\
& 30 & $403.23 \pm 36.86$ & $0.23 \pm 0.031$ & $80.66 \pm 6.54$ & $0.88 \pm 0.035$ \\
& 46 & $458.88 \pm 32.48$ & $0.25 \pm 0.041$ & $81.34 \pm 7.03$ & $0.86 \pm 0.041$ \\
WKY & 14 & $358.18 \pm 78.02$ & $0.20 \pm 0.026$ & $48.24 \pm 5.71^{\mathrm{a}}$ & $0.41 \pm 0.055^{\mathrm{a}}$ \\
& 30 & $385.23 \pm 41.43$ & $0.20 \pm 0.029$ & $41.64 \pm 5.79^{\mathrm{a}}$ & $0.45 \pm 0.041^{\mathrm{a}}$ \\
& 46 & $367.71 \pm 43.69^{\mathrm{a}}$ & $0.21 \pm 0.019^{\mathrm{a}}$ & $41.70 \pm 5.02^{\mathrm{a}}$ & $0.42 \pm 0.071^{\mathrm{a}}$ \\
SHR-Los & 14 & $485.18 \pm 94.25^{\mathrm{a}, \mathrm{b}}$ & $0.13 \pm 0.021^{\mathrm{a}, \mathrm{b}}$ & $50.90 \pm 6.61^{\mathrm{a}}$ & $0.57 \pm 0.035^{\mathrm{a}, \mathrm{b}}$ \\
& 30 & $319.02 \pm 53.78^{\mathrm{a}, \mathrm{b}}$ & $0.15 \pm 0.020^{\mathrm{a}, \mathrm{b}}$ & $57.21 \pm 4.17^{\mathrm{a}, \mathrm{b}}$ & $0.62 \pm 0.042^{\mathrm{a}, \mathrm{b}}$ \\
SHR-Aml & 46 & $302.11 \pm 32.36^{\mathrm{a}, \mathrm{b}}$ & $0.17 \pm 0.020^{\mathrm{a}, \mathrm{b}}$ & $57.70 \pm 6.31^{\mathrm{a}, \mathrm{b}}$ & $0.57 \pm 0.056^{\mathrm{a}, \mathrm{b}}$ \\
& 14 & $478.92 \pm 73.99^{\mathrm{a}, \mathrm{b}}$ & $0.19 \pm 0.019$ & $84.75 \pm 4.96^{\mathrm{b}, \mathrm{c}}$ & $0.76 \pm 0.071^{\mathrm{b}, \mathrm{c}}$ \\
& 30 & $391.39 \pm 40.56^{\mathrm{c}}$ & $0.22 \pm 0.036^{\mathrm{c}}$ & $76.89 \pm 3.31^{\mathrm{a}-\mathrm{c}}$ & $0.82 \pm 0.022^{\mathrm{a}-\mathrm{c}}$
\end{tabular}

${ }^{a} \mathrm{P}<0.05$ vs. SHRs at the same age; ${ }^{\mathrm{b}} \mathrm{P}<0.05$ vs. WKYs at the same age; ${ }^{\mathrm{C}} \mathrm{P}<0.05$ vs. SHR-Los at the same age. The data are represented as the means $\pm \mathrm{SD}$.

higher compared to that in WKYs $(\mathrm{P}<0.05)$, the cardiac BNP mRNA in SHR-Los was observably decreased as compared with untreated SHRs, but was still higher compared to that in
WKYs $(\mathrm{P}<0.05)$ (Fig. 7). In contrast, the cardiac BNP mRNA expression in SHR-Aml was slightly and significantly increased compared with untreated SHRs $(\mathrm{P}<0.05)$. 

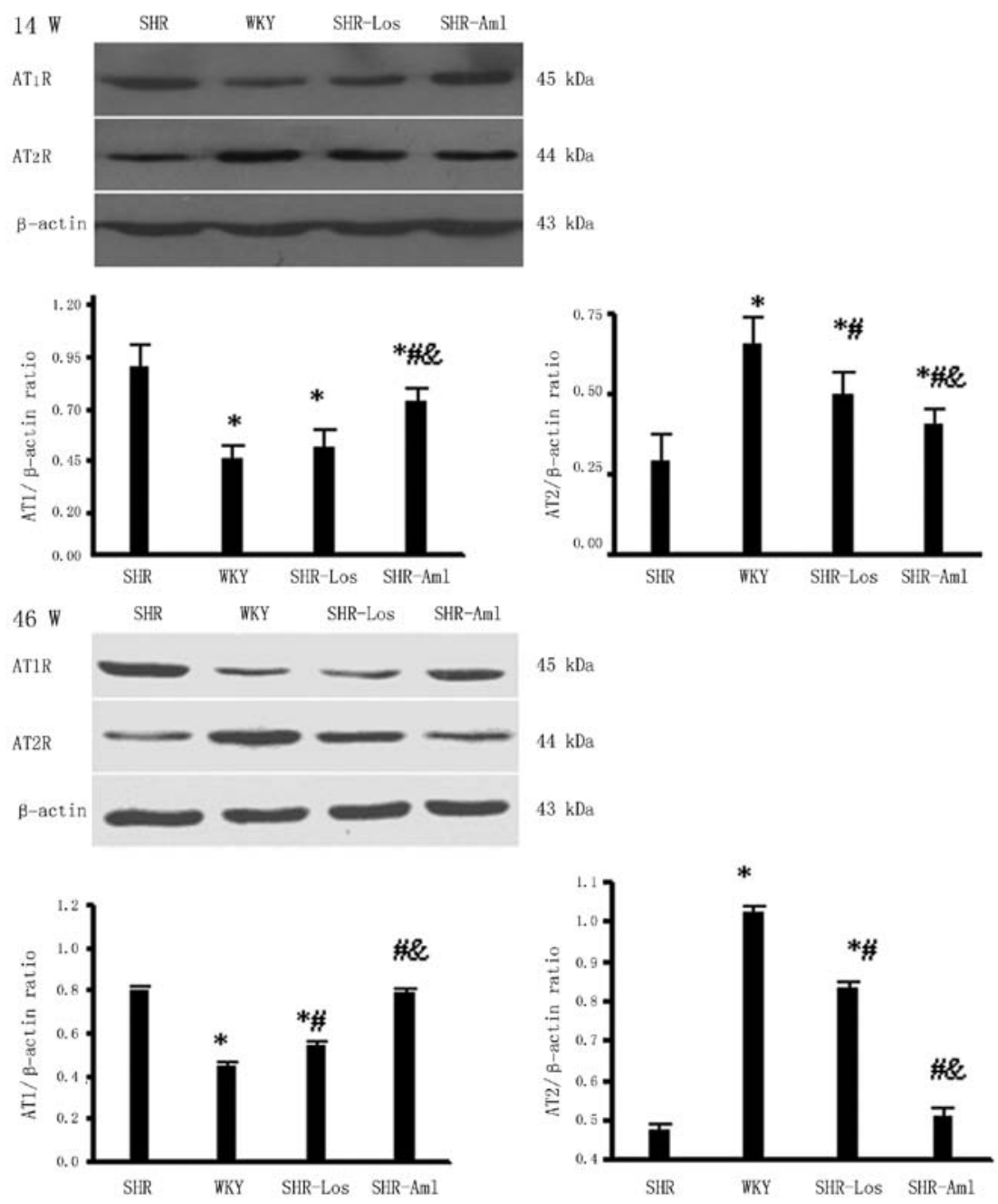

Figure 5. Western blotting of $\mathrm{AT}_{1} \mathrm{R}$ and $\mathrm{AT}_{2} \mathrm{R}$ in myocardium of 14- and 46-week-old WKYs, and untreated and prehypertensively treated $\mathrm{SHR}_{\mathrm{N}} \mathrm{AT}_{1} \mathrm{R}$, angiotensin II type 1 receptor; $\mathrm{AT}_{2} \mathrm{R}$, angiotensin II type 2 receptor. ${ }^{*} \mathrm{P}<0.05$ vs. SHR; ${ }^{\mathrm{P}} \mathrm{P}<0.05$ vs. WKY; ${ }^{\circledR} \mathrm{P}<0.05$ vs. SHR-Los.

\section{Discussion}

In the present study, we demonstrated that transient prehypertensive treatment with losartan was more effective than amlodipine in delaying a long-term blood pressure (BP) increase and ameliorating renal, cardiac structure and function, and that the mechanism responsible for losartan's superiority may be due to its permanent attenuation of both circulating and local renin-angiotensin systems (RAS).

Our results demonstrated that although the antihypertensive effects were similar between the two therapeutic regimens during treatment, BP development differed significantly after drug withdrawal. BP in losartan-treated SHRs (SHR-Los) remained significantly lower compared to that in untreated SHRs until week 46, whereas BP in amlodipine-treated SHRs (SHR-Aml) returned to untreated SHR levels from 30 weeks onwards. This finding notably coincides with a previous study by Christensen et al (18) that SHR treatment with angiotensinconverting-enzyme inhibitor (ACEI) rather than $\beta$-blocker, vasodilator or calcium antagonist prevented BP increase after withdrawal of long-term treatment. Whether or not the ACEI or angiotensin-receptor blockers (ARB) had longer effects on $\mathrm{BP}$ reduction compared to other forms of therapies remains unclear. One possibility is related to the 'RAS block memory' via suppression of a 'reno-vascular amplifier' mechanism (19-21). According to this hypothesis, during the critical period of hypertension development in juveniles, increased BP leads to vascular hypertrophy in the resistance vessels, which decreases glomerular perfusion and results in increased synthesis of renin and activation of RAS. These changes cause a further BP increase and initiate a positive feedback loop. RAS inhibitors may block this vicious cycle by attenuating the rise in BP and importantly, by decreasing the vascular hypertrophy. Other possibilities are that alterations in the central RAS, decreases in plasma AVP levels and alterations in endothelial function may be involved in the prolonged hypotensive effect (22-24). Recently Baumann et al (25) demonstrated that a BP decrease after transient ARB is associated with an increased medullary blood flow and a reduced medullary pericyte number. These possibilities are not mutually exclusive, and it is possible that multiple mechanisms may be involved in the sustained suppression of BP. As to the prolonged pressure-lowering effects of amlodipine, BP development after drug withdrawal in our study was different from the result reported by Sevilla et al (26). They indicated that treatments of adult SHRs with 8 and $20 \mathrm{mg} / \mathrm{kg} / \mathrm{day}$ of amlodipine for six months yielded 


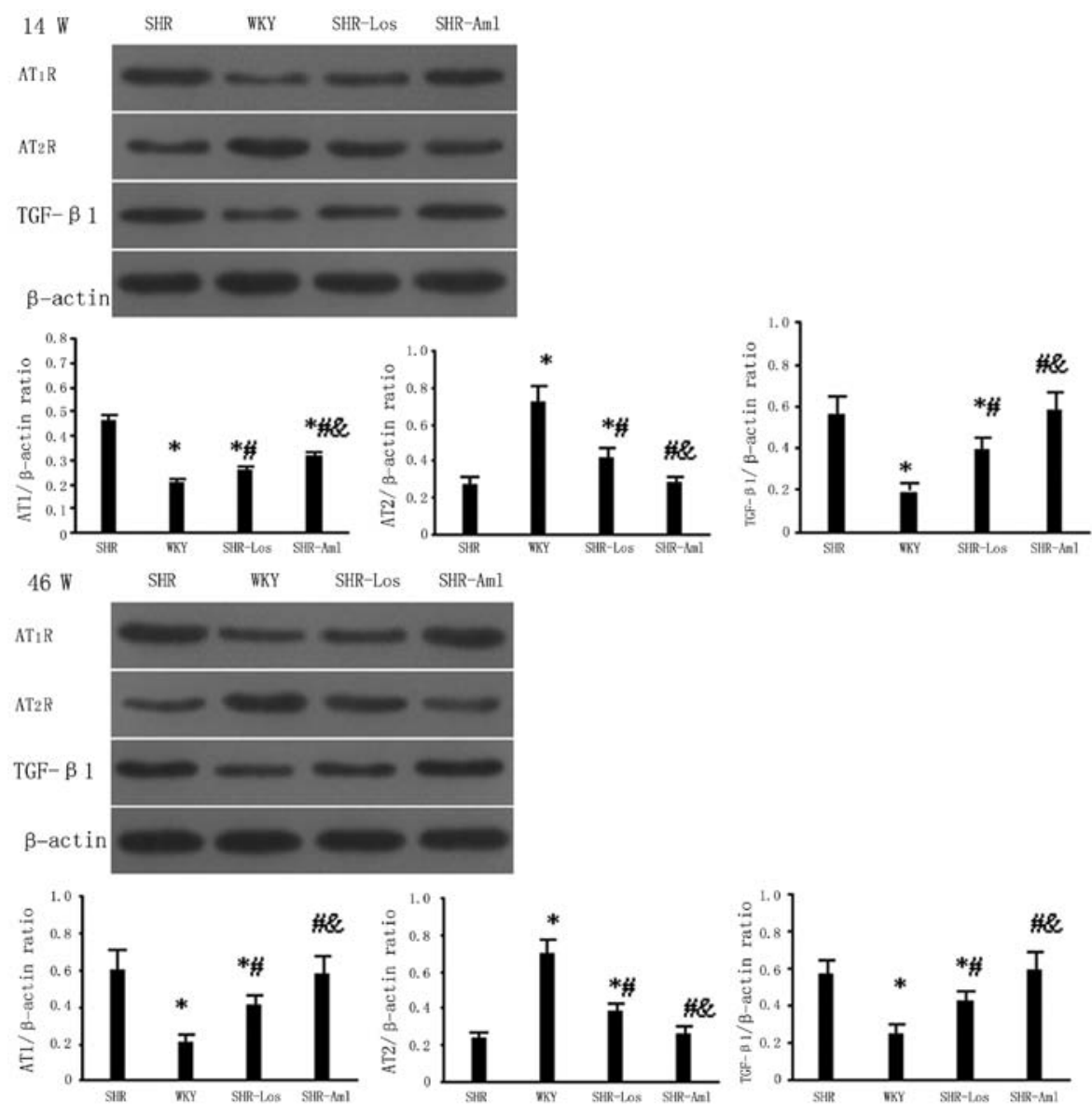

Figure 6. Representative western blotting autoradiogram of $\mathrm{AT}_{1} \mathrm{R}, \mathrm{AT}_{2} \mathrm{R}, \mathrm{TGF}-\beta_{1}$ protein in renal cortex tissue of 14- and 46-week-old WKYs, and untreated and prehypertensively treated SHRs. ${ }^{*} \mathrm{P}<0.05$ vs. SHR; ${ }^{*} \mathrm{P}<0.05$ vs. WKY; ${ }^{\circledR} \mathrm{P}<0.05$ vs. SHR-Los.
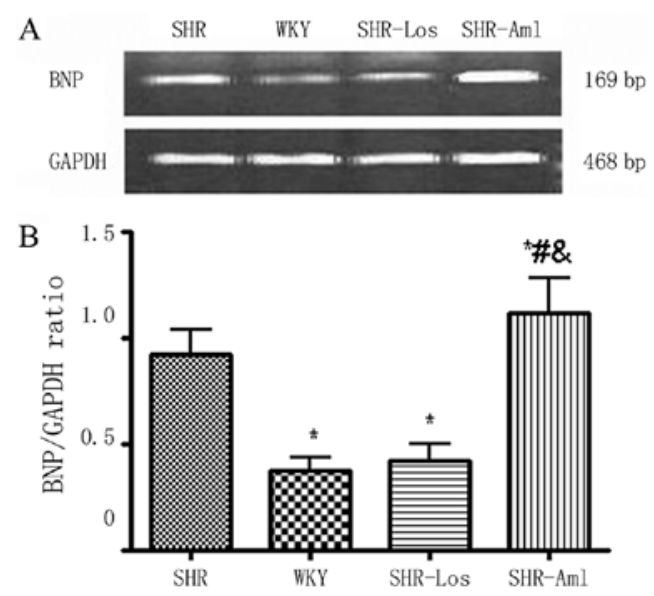

Figure 7. Comparison of cardiac BNP mRNA expression in 46-week-old WKYs, untreated and prehypertensively treated SHRs. BNP, brain natriuretic peptide; GAPDH, glyceraldehyde phosphate dehydrogenase. "P<0.05 vs. SHR; ${ }^{\text {"}} \mathrm{P}<0.05$ vs. WKY; ${ }^{\mathrm{P}} \mathrm{P}<0.05$ vs. SHR-Los.

persistent hypotensive effects even at three months after drug withdrawal. A potential reason for this difference was that amlodipine treatment was administered too early and for a limited time period to reverse vascular hypertrophy and break the vicious cycle.

With respect to the effects on cardiac protection, our results demonstrated that prehypertensive treatment with losartan permanently reduced cardiac hypertrophy and fibrosis until week 46. Longitudinal echocardiographic evaluation revealed identical values of interventricular septum thickness (IVSTd) between SHR-Los and WKYs, and the left ventricular end-diastolic dimension (LVEDD) remained stable over the follow-up period. Besides these, the left ventricular ejection fraction (LVEF) and cardiac BNP mRNA expression were improved in SHR-Los compared with untreated SHRs at week 46. All the above changes suggest prolonged cardioprotective effects by losartan treatment. The mechanism to determine how transient prehypertensive treatment with losartan exerts sustained cardiac protection is required. Previous studies have mainly explained the mechanism through the reduction of gene expression of cardiac Ang II type 1 receptor $\left(\mathrm{AT}_{1} \mathrm{R}\right)(10)$ or plasma Ang II concentration (27) and not by a BP decrease alone. In our study, we measured the circulating and local angiotensin II and Aldo levels by radioimmunoassay and discovered that, besides the suppressant effects on circulating RAS (at week 14, the plasma Ang II was elevated via negative feedback regulation), the cardiac RAS was also significantly inhibited, which has been proven to be involved in the pathogenesis of cardiac hypertrophy and fibrosis $(28,29)$. Furthermore, western blot analysis detected a favorably altered $\mathrm{AT}_{1} \mathrm{R} / \mathrm{AT}_{2} \mathrm{R}$ balance with losartan treatment, which consisted of downregulated cardiac $\mathrm{AT}_{1} \mathrm{R}$ protein but upregulated $\mathrm{AT}_{2} \mathrm{R}$ protein. Although how low levels of local Ang II act on high levels of $\mathrm{AT}_{2} \mathrm{R}$ to 
ameliorate cardiac damage remains unknown, our results revealed that transient prehypertensive treatment with losartan may maintain the circulating and local RAS in an initial low-activity or physiologic state. Unlike the results reported by Baumann et al (10) the SBP in our study remained lower compared to the untreated SHRs during the experimental period; thus, it cannot be precluded that the beneficial effects of losartan treatment is related to a BP decrease alone.

Noteworthy, the LVMI in amlodipine-treated SHR was similar to that in untreated SHRs at weeks 14 and 46, but was significantly decreased at week 30 . The possible reason for this phenomenon is that the cardiac hypertrophy of SHRs is fully established between week 14 and 30 (30), and amlodipine treatment causes hypertrophy regression via its residual pressure reduction during this period and the antihypertrophy action tapers with the disappearance of antihypertensive effects from 30 weeks onwards. Unexpectedly, the SHR-Aml exhibited no difference in CVF from untreated SHRs at weeks 14 and 30, but an observably higher value compared to the untreated SHRs at week 46. Moreover, the echocardiography displayed enlarged LVEDD and declined EF at the end of follow-up, and RT-PCR assay detected a slightly and significantly increased expression of BNP mRNA. These results implied a detrimental effect of amlodipine treatment, which we speculated may be related to the negative inotropic effects of amlodipine since the plasma and myocardium Ang II and Aldo levels were comparable between SHR-Aml and untreated SHRs at week 46. Our speculation contradicted the commonly accepted properties of amlodipine of insignificant negative inotropic effects (31). The specific intervention period or inappropriate dose of amlodipine in our study may be responsible for this discrepancy, yet a detailed mechanism needs to be further investigated.

Circulating RAS regulates physiological responses, whereas the local RAS is activated during tissue injury and contributes to pathological processes, including cell proliferation/apoptosis, fibrosis and inflammation. Ang II is one key factor in the development of renal fibrosis. In this study, our data demonstrated that transient prehypertensive treatment with losartan reduces Ang II and Aldo concentrations both in plasma and renal tissue, upregulates $\mathrm{AT}_{2} \mathrm{R}$ protein expression while downregulating $\mathrm{AT}_{1} \mathrm{R}$ protein expression. It revealed that transient prehypertensive treatment with losartan may maintain the circulating and local RAS in a low-activity state. Furthermore, $\mathrm{AT}_{2} \mathrm{R}$ upregulation in SHR-Los inhibits progress of glomerulosclerosis, supporting key effects of $\mathrm{AT}_{1} \mathrm{R} / \mathrm{AT}_{2} \mathrm{R}$ balance in fibrotic states. Although regulation of the expression of the $\mathrm{AT}_{2} \mathrm{R}$ is poorly understood, previous studies have proven the $\mathrm{AT}_{2} \mathrm{R}$ actions contribute to $\mathrm{ARB}$ effects in the kidney (32).

In summary, our results suggest that transient $\mathrm{AT}_{1} \mathrm{R}$ blockade in young SHRs is superior to the calcium antagonist on delaying long-term BP rise and ameliorating cardiac, renal structure and function, which corroborated the effectiveness and feasibility of an RAS inhibitor for prehypertensive treatment demonstrated by TROPHY and PHARAO trials. However, the superiority of ARB over a calcium channel blocker (CCB) observed in animal experiments should be cautiously interpreted when translated into clinical settings, since the prehypertensive condition of human beings is far more complicated (e.g., strain difference, attendant obesity, dyslipidemia or impaired glucose tolerance) as compared with SHRs. Moreover, since the recently published trial of DHYPP demonstrated that one year of candesartan treatment in subjects with high familial risk of future hypertension had no persistent effect on BP when treatment was discontinued (33), further studies to explore how to 'apply' the prolonged cardioprotective effects observed in animals to human beings is required. Nevertheless, our data have important clinical implications that ARB may be a better choice compared with $\mathrm{CCB}$ for hypertension prevention and cardiac protection.

\section{Acknowledgements}

This study was supported by a grant from the National Natural Science Foundation of China (81070207). Amlodipine was generously supplied by Pfizer Pharmaceutical, Inc. The authors would like to thank Meiyan Lin and Shusheng Liao for their technical support.

\section{References}

1. Vasan RS, Larson MG, Leip EP, Kannel WB and Levy D: Assessment of frequency of progression to hypertension in non-hypertensive participants in the Framingham Heart Study: a cohort study. Lancet 358: 1682-1686, 2001.

2. Hsia J, Margolis KL, Eaton CB, et al: Prehypertension and cardiovascular disease risk in the Women's Health Initiative. Circulation 115: 855-860, 2007.

3. Knecht S, Wersching H, Lohmann $\mathrm{H}$, et al: High-normal blood pressure is associated with poor cognitive performance. Hypertension 51: 663-668, 2008.

4. Mullican DR, Lorenzo C and Haffner SM: Is prehypertension a risk factor for the development of type 2 diabetes? Diabetes Care 32: $1870-1872,2009$

5. Ninomiya T, Kubo M, Doi Y, et al: Prehypertension increases the risk for renal arteriosclerosis in autopsies: the Hisayama Study. J Am Soc Nephrol 18: 2135-2142, 2007.

6. Chobanian AV, Bakris GL, Black HR, et al: The Seventh Report of the Joint National Committee on Prevention, Detection, Evaluation, and Treatment of High Blood Pressure: the JNC 7 report. JAMA 289: 2560-2572, 2003.

7. Julius S, Nesbitt SD, Egan BM, et al: Feasibility of treating prehypertension with an angiotensin-receptor blocker. N Engl J Med 354: 1685-1697, 2006.

8. Luders S, Schrader J, Berger J, et al: The PHARAO study: prevention of hypertension with the angiotensin-converting enzyme inhibitor ramipril in patients with high-normal blood pressure: a prospective, randomized, controlled prevention trial of the German Hypertension League. J Hypertens 26: 1487-1496, 2008.

9. Harrap SB, Van der Merwe WM, Griffin SA, Macpherson F and Lever AF: Brief angiotensin converting enzyme inhibitor treatment in young spontaneously hypertensive rats reduces blood pressure long-term. Hypertension 16: 603-614, 1990.

10. Baumann M, Janssen BJ, Hermans JJ, et al: Transient AT1 receptor-inhibition in prehypertensive spontaneously hypertensive rats results in maintained cardiac protection until advanced age. J Hypertens 25: 207-215, 2007.

11. Baumann M, Hermans JJ, Janssen BJ, et al: Transient prehypertensive treatment in spontaneously hypertensive rats: a comparison of spironolactone and losartan regarding long-term blood pressure and target organ damage. J Hypertens 25: 2504-2511, 2007.

12. Tomassoni D, Sabbatini M and Amenta F: Effect of different dihydropyridine-type $\mathrm{Ca}^{2+}$ antagonists on left ventricle hypertrophy and coronary changes in spontaneously hypertensive rats. J Cardiovasc Pharmacol 41: 544-552, 2003.

13. Watanabe M, Kawaguchi H, Onozuka H, et al: Chronic effects of enalapril and amlodipine on cardiac remodeling in cardiomyopathic hamster hearts. J Cardiovasc Pharmacol 32: 248-259, 1998. 
14. Widdop RE and Li XC: A simple versatile method for measuring tail cuff systolic blood pressure in conscious rats. Clin Sci (Lond) 93: 191-194, 1997.

15. Lang RM, Bierig M, Devereux RB, et al: Recommendations for chamber quantification: a report from the American Society of Echocardiography's Guidelines and Standards Committee and the Chamber Quantification Writing Group, developed in conjunction with the European Association of Echocardiography, a branch of the European Society of Cardiology. J Am Soc Echocardiogr 18: 1440-1463, 2005.

16. Floege J, Hackmann B, Kliem V, et al: Age-related glomerulosclerosis and interstitial fibrosis in Milan normotensive rats: a podocyte disease. Kidney Int 51: 230-243, 1997.

17. Xu Y, Kumar D, Dyck JR, et al: AT(1) and AT(2) receptor expression and blockade after acute ischemia-reperfusion in isolated working rat hearts. Am J Physiol Heart Circ Physiol 282: H1206-H1215, 2002.

18. Christensen KL, Jespersen LT and Mulvany MJ: Development of blood pressure in spontaneously hypertensive rats after withdrawal of long-term treatment related to vascular structure. J Hypertens 7: 83-90, 1989.

19. Ishiguro K, Sasamura H, Sakamaki Y, Itoh H and Saruta T: Developmental activity of the renin-angiotensin system during the 'critical period' modulates later L-NAME-induced hypertension and renal injury. Hypertens Res 30: 63-75, 2007.

20. Nakaya H, Sasamura H, Mifune M, et al: Prepubertal treatment with angiotensin receptor blocker causes partial attenuation of hypertension and renal damage in adult Dahl salt-sensitive rats. Nephron 91: 710-718, 2002.

21. Sasamura H, Hayashi K, Ishiguro K, Nakaya $H$, Saruta $T$ and Itoh $\mathrm{H}$ : Prevention and regression of hypertension: role of renal microvascular protection. Hypertens Res 32: 658-664, 2009.

22. Keaton AK, White CR and Berecek KH: Captopril treatment and its withdrawal prevents impairment of endotheliumdependent responses in the spontaneously hypertensive rat. Clin Exp Hypertens 20: 847-866, 1998.

23. Zhang L, Edwards DG and Berecek KH: Effects of early captopril treatment and its removal on plasma angiotensin converting enzyme (ACE) activity and arginine vasopressin in hypertensive rats (SHR) and normotensive rats (WKY). Clin Exp Hypertens 18: 201-226, 1996.
24. Wu JN and Berecek KH: Prevention of genetic hypertension by early treatment of spontaneously hypertensive rats with the angiotensin converting enzyme inhibitor captopril. Hypertension 22: 139-146, 1993

25. Baumann M, Janssen BJ, Rob Hermans JJ, Bartholome R, Smits JF and Struijker Boudier HA: Renal medullary effects of transient prehypertensive treatment in young spontaneously hypertensive rats. Acta Physiol (Oxf) 196: 231-237, 2009.

26. Sevilla MA, Voces F, Carron R, et al: Amlodipine decreases fibrosis and cardiac hypertrophy in spontaneously hypertensive rats: persistent effects after withdrawal. Life Sci 75: 881-891, 2004.

27. Takemori $\mathrm{K}$, Ishida $\mathrm{H}$ and Ito $\mathrm{H}$ : Continuous inhibition of the renin-angiotensin system and protection from hypertensive end-organ damage by brief treatment with angiotensin II type 1 receptor blocker in stroke-prone spontaneously hypertensive rats. Life Sci 77: 2233-2245, 2005.

28. Bader M: Role of the local renin-angiotensin system in cardiac damage: a minireview focussing on transgenic animal models. J Mol Cell Cardiol 34: 1455-1462, 2002.

29. Mizuno K, Tani M, Hashimoto S, et al: Effects of losartan, a nonpeptide angiotensin II receptor antagonist, on cardiac hypertrophy and the tissue angiotensin II content in spontaneously hypertensive rats. Life Sci 51: 367-374, 1992.

30. Adams MA, Bobik A and Korner PI: Differential development of vascular and cardiac hypertrophy in genetic hypertension. Relation to sympathetic function. Hypertension 14: 191-202, 1989.

31. Burges R and Moisey D: Unique pharmacologic properties of amlodipine. Am J Cardiol 73: 2A-9A, 1994.

32. Naito T, Ma LJ, Yang H, et al: Angiotensin type 2 receptor actions contribute to angiotensin type 1 receptor blocker effects on kidney fibrosis. Am J Physiol Renal Physiol 298: F683-F691, 2010.

33. Skov K, Eiskjaer H, Hansen HE, Madsen JK, Kvist S and Mulvany MJ: Treatment of young subjects at high familial risk of future hypertension with an angiotensin-receptor blocker. Hypertension 50: 89-95, 2007. 